
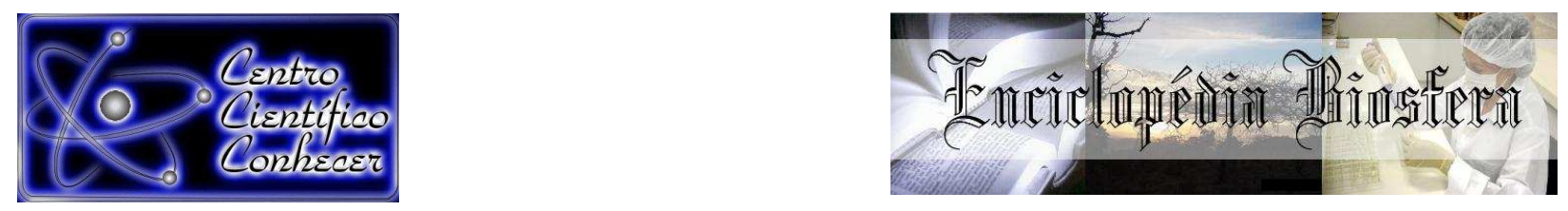

\title{
AVALIAÇÃO DA POLUIÇÃO SONORA EM UMA UNIVERSIDADE DA CIDADE DE SÃO CARLOS - SP
}

\author{
Tatiane Tagino Comin ${ }^{1}$, Mônica Lopes Aguiar² \\ 1 Doutoranda em Engenharia Química da Universidade Federal de São Carlos \\ (tati.comin@gmail.com) São Paulo - Brasil \\ 2 Prof Doutora do Departamento de Engenharia Química da Universidade Federal \\ de São Carlos \\ Recebido em: 08/04/2016 - Aprovado em: 30/05/2016 - Publicado em: 20/06/2016 \\ DOI: 10.18677/Enciclopedia_Biosfera_2016_145
}

\begin{abstract}
RESUMO
A poluição sonora é tratada como um problema de saúde pública pela Organização Mundial de Saúde desde a década de 1980, porém inicialmente as pesquisas sobre o tema eram restritas a ambientes industriais. Comprovações científicas que os efeitos deletérios da exposição à poluição sonora não estão apenas relacionados com o aparelho auditivo humano, podendo ocasionar desde dificuldades de concentração até alterações bioquímicas e cardiovasculares, impulsionaram estudos em outros locais e o ambiente escolar tem despertado especial interesse desde então. Neste contexto, o objetivo deste estudo foi avaliar os níveis de poluição sonora na Universidade Federal de São Carlos (SP). A coleta de dados foi realizada de maneira simultânea nos ambientes internos e externos de quatro salas de aula selecionadas e os níveis de pressão sonora foram monitorados com decibelímetros digitais (modelo DEC-490, marca Instrutherm) e, posteriormente, foram comparados com os padrões estabelecidos pela NBR 10.152/1987 da Associação Brasileira de Normas Técnicas. Verificou-se que em todas as salas de aula os níveis médios de ruídos ultrapassaram $50 \mathrm{~dB}$, valor máximo tolerado pela $A B N T$, indicando que a poluição sonora existe em níveis alarmantes nesta universidade e que é importante a adoção de medidas corretivas e preventivas para mitigar esta situação.
\end{abstract}

PALAVRAS-CHAVE: limites de emissão, ruídos, salas de aula.

\section{EVALUATION OF NOISE POLLUTION IN CLASSROOM ENVIRONMENTS IN THE CITY OF SÃO CARLOS - SP}

\begin{abstract}
Noise pollution has been treated as a public health problem by the World Health Organization since the 1980s, although research on the issue was initially restricted to industrial environments. Scientific evidence suggesting that the negative effects of exposure to noise pollution are not only associated with the human auditory apparatus, but can also cause difficulties in concentration and even biochemical and cardiovascular alterations, has stimulated studies in other locations, and the classroom environment has attracted particular attention. The aim of this study was to evaluate the levels of noise pollution at the Federal University of São Carlos (SP). Data collection was performed simultaneously in the internal and external environments of four selected classrooms, with sound levels monitored using digital decibel meters (Model DEC-490, Instrutherm). The values were then compared with
\end{abstract}


the limit values defined in the NBR 10.152/1987 norm of the Brazilian Technical Standards Association (ABNT). It was found that in all the classrooms, the average noise levels exceeded $50 \mathrm{~dB}$, the maximum value permitted by ABNT, indicating that there were high levels of noise pollution at the university, and that corrective and preventive measures should be implemented to improve this situation.

KEYWORDS: sound, classrooms, emission limits.

\section{INTRODUÇÃO}

Poluição sonora pode ser definida como "som indesejado ou perturbador", segundo a Agência de Proteção Ambiental dos Estados Unidos (ENVIRONMENTAL PROTECTION AGENCY, 2016). O som torna-se indesejável quando interfere nas atividades normais de um ser humano, comprometendo sua qualidade de vida. Ruído, por sua vez, pode fisicamente ser definido como fenômeno acústico não periódico, sem componentes harmônicos definidos, ou seja, compreende uma mistura de sons, cujas frequências não possuem uma periodicidade.

Em 1980, a Organização Mundial de Saúde (OMS) reconheceu a poluição sonora como um problema de saúde pública e alertou que a exposição a ruídos pode ocasionar danos à audição, distúrbios do sono, diminuição do rendimento no trabalho ou escola, entre outros. A OMS, desde então, atua para nortear os padrões de emissão de ruídos para garantir o bem-estar da população (TORRES et al., 2013).

Nas décadas seguintes, estudos sobre a poluição sonora concluíram que os efeitos ambientais do ruído dependem não só da energia total (medida em decibéis), mas também da altura, frequência, padrão de tempo e duração da exposição (BOTKIN \& KELLER, 2011). O ouvido humano pode suportar sons de até $60 \mathrm{~dB}$ sem danos ou perda auditiva. Porém, qualquer ruído acima de $80 \mathrm{~dB}$ é potencialmente perigoso, pois a exposição por tempo prolongado pode provocar alterações cardiovasculares (como o aumento da pressão arterial) e bioquímicas (como oscilações na produção de glicose e de cortisona), interferir na resposta vegetativa (ocasionando desde dilatação das pupilas até mudanças gastrointestinais), além de efeitos sociológicos (como perturbação do sono e prejuízos na concentração e performance). As vibrações produzidas por ruídos podem afetar a parede torácica provocando alterações no ritmo respiratório e também comprometer o senso de equilíbrio (SILVA et al., 2015).

As pesquisas científicas pioneiras na temática de ruídos eram restritas a ambientes industriais, pois nos chamados "chão de fábricas" que eram observados danos físicos em curto prazo, especialmente aqueles relacionados à perda de audição. Atualmente, o tema se expandiu e ambientes, antes ignorados, conquistaram a atenção de pesquisadores como é o caso de escolas e universidades.

$\mathrm{Na}$ cidade de São Carlos (SP), dois importantes estudos sobre poluição sonora em ambientes escolares foram efetuados na última década. No ano de 2008, o monitoramento de duas salas de aula da Universidade de São Paulo (Campus São Carlos, Prédio 2) indicou níveis de ruídos entre 50 e 62 dB e, em 2012, e a avaliação de uma escola estadual da região central da cidade registrou níveis internos entre 58 e $75 \mathrm{~dB}$ (COMIN, 2016). Ambos os trabalhos alertaram que o ruído era uma variável ambiental que merecia atenção, demandando a necessidade de medidas corretivas e preventivas, pois seus níveis extrapolaram o limite máximo de $50 \mathrm{~dB}$ previsto, pela Norma Brasileira (NBR) 10.152/1987 da ABNT (ASSOCIAÇÃO BRASILEIRA DE NORMAS TÉCNICAS, 1987), para salas de aula. 
Neste contexto, objetivou-se avaliar a poluição sonora, por meio do monitoramento dos níveis de ruídos, nos interiores e exteriores de quatro salas de aula da Universidade Federal de São Carlos (SP), possibilitando comparar com as normas vigentes no Brasil e, quando estiveram em desacordo, sugerir medidas corretivas e preventivas para mitigar os níveis elevados.

\section{MATERIAL E MÉTODOS}

Para este estudo, selecionou-se a Universidade Federal de São Carlos (UFSCar), uma das instituições públicas de ensino superior da cidade, que, além do grande fluxo de pessoas (mais de 15.000 entre alunos, professores e demais servidores), fica localizada na Rodovia Washington Luís (SP-310, Km 235) onde há intenso tráfego veicular.

$\mathrm{Na}$ UFSCar, foram selecionadas quatro salas de aula em prédios situados na "área norte" da instituição, próximas à Biblioteca, por onde circulam milhares de pessoas diariamente. Duas destas salas de aula possuíam apenas ventilação natural (por meio apenas de portas e janelas, sem nenhum meio mecânico) e nas outras duas havia aparelho de ar condicionado instalado (ambos do tipo split pisoteto, modelo GST36-22L/A da marca Gree). Para cada sala foram definidos dois pontos amostrais, um interno (entre a lousa, mesa do professor e primeira fileira de carteiras) e outro externo, fixado no pátio lateral de cada sala, pois o monitoramento simultâneo destes ambientes é fundamental para critérios de comparação. Assim, os ambientes selecionados foram monitorados no horário regular de aulas, das 8 às 18 horas, sendo que cada local foi avaliado quatro vezes, durante quatro meses.

\section{Monitoramento de ruídos}

Para nortear o monitoramento de ruídos foram adotadas as normas NBR 10.151/2000 e NBR 10.152/1987 da ABNT (ASSOCIAÇÃO BRASILEIRA DE NORMAS TÉCNICAS, 1987; 2000). Relevou-se a recomendação para uso de equipamento adequado para medir o nível de pressão sonora equivalente ponderado em "A", expresso na escala dBA, considerada apropriada para representar a resposta humana para sons de, aproximadamente, 55 decibéis. Especificamente para o interior de salas de aula, a faixa considerada adequada pela ABNT é de 40 a $50 \mathrm{~dB}$, enquanto para circulação externa em ambientes escolares é de 45 a $55 \mathrm{~dB}$.

Assim, optou-se pela utilização de decibelímetros digitais, modelo DEC-490 (Instrutherm), com resolução de $0,1 \mathrm{~dB}$ e precisão de $\pm 1,4 \mathrm{~dB}$, selecionado a faixa de medição entre 30 e 130 dBA. Foram construídos dois suportes metálicos para comportar os decibelímetros há 1,5 metros do solo, altura considerada adequada segundo as normas adotadas (Figura 1). Cabe enfatizar que um suporte era deixado dentro da sala de aula e o outro no exterior, no pátio lateral. 


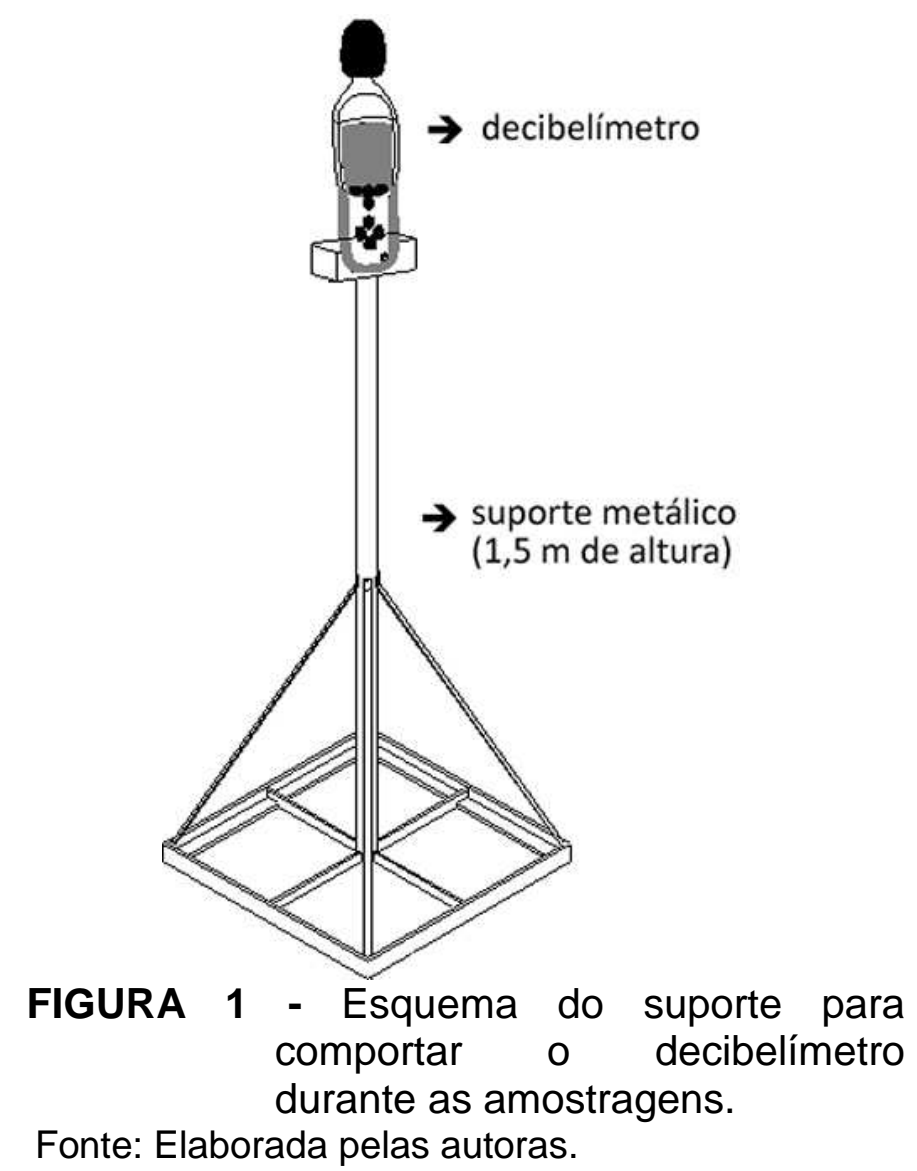

As medições foram realizadas em duas etapas: na primeira avaliaram-se os níveis de ruídos com as salas de aula desocupadas e na segunda nas condições normais de utilização das salas, ou seja, com a presença de alunos e professores, respeitando-se a rotina de aulas e monitorando-se simultaneamente os respectivos pátios laterais externos.

Posteriormente, estes dados foram analisados utilizando-se ferramentas de Estatística Descritiva. Adotaram-se as médias aritméticas como medida de tendência central e como medida de dispersão foram determinados os desvios-padrão. Foram também obtidas as razões entre os níveis internos e externos, denominadas de "índices l/E".

\section{RESULTADOS E DISCUSSÃO}

A primeira etapa do monitoramento consistiu em verificar os níveis de ruídos nas salas de aula desocupadas. Como o decibelímetro armazena os dados a cada 30 segundos foram calculadas as médias aritméticas para cada local e os resultados encontram-se na Tabela 1, cujas abreviaturas "VN" $\mathrm{e}$ "AC" significam, respectivamente, ventilação natural e ar-condicionado. É importante citar que nas salas climatizadas, o ar-condicionado foi mantido ligado, para verificar o quanto seu funcionamento normal contribuía para a poluição sonora local e nas salas com ventilação natural, portas e janelas permaneceram abertas durante o experimento. 
TABELA 1 - Médias para os níveis de ruídos nas salas vazias

\begin{tabular}{cc}
\hline Salas de aula & Médias para ruídos (dBA) \\
\hline A (AC) & 47,2 \\
B (AC) & 52,8 \\
C (VN) & 44,5 \\
D (VN) & 38,6 \\
\hline
\end{tabular}

AC- Ar condicionado; VN - Ventilação Natural

Inicialmente constatou-se que as salas que possuíam aparelhos de arcondicionado instalados apresentaram níveis médios superiores aos das salas com ventilação natural. Isto mostra a relevante contribuição dos ruídos gerados pelo próprio funcionamento dos aparelhos de ar condicionado, infringindo a faixa permissível de 35 a $45 \mathrm{dBA}$ prevista para salas de aula vazias que possuam sistema de condicionamento de ar instalado, conforme a NBR 6401 da ABNT (ASSOCIAÇÃO BRASILEIRA DE NORMAS TÉCNICAS, 1980).

$\mathrm{Na}$ etapa seguinte, cada sala de aula e seu respectivo pátio lateral externo foram monitorados quatro vezes, uma vez por semana. A Tabela 2 apresenta a média aritmética global para cada sala, desvios padrão e índices I/E (quociente entre os níveis médios internos e externos) para os dados de pressão sonora, que indicam o nível de ruído no decorrer das aulas.

TABELA 2 - Resultados dos níveis de pressão sonora monitorados na UFSCar

\begin{tabular}{|c|c|c|c|c|c|}
\hline \multirow[b]{2}{*}{ Local } & \multicolumn{2}{|c|}{ Dados internos } & \multicolumn{2}{|c|}{ Dados externos } & \multirow{2}{*}{$\begin{array}{c}\text { Índices } \\
\text { I/E }\end{array}$} \\
\hline & $\begin{array}{l}\text { Média } \\
\text { (dBA) }\end{array}$ & $\begin{array}{l}\text { Desvio } \\
\text { padrão }\end{array}$ & $\begin{array}{l}\text { Média } \\
\text { (dBA) }\end{array}$ & $\begin{array}{l}\text { Desvio } \\
\text { padrão }\end{array}$ & \\
\hline Sala A (AC) & 63,4 & 7,0 & 56,4 & 3,9 & 1,12 \\
\hline Sala B (AC) & 59,2 & 5,4 & 55,3 & 3,1 & 1,07 \\
\hline Sala C (VN) & 56,6 & 4,1 & 60,5 & 3,2 & 0,94 \\
\hline Sala D (VN) & 57,4 & 6,6 & 57,5 & 4,7 & 1,00 \\
\hline
\end{tabular}

AC- Ar condicionado; VN - Ventilação Natural

$\mathrm{Na}$ Tabela 2 verifica-se que nas duas salas que possuíam ar-condicionado as médias internas foram maiores que as externas, resultando em índices l/E superiores a 1,0. Em contrapartida, nas salas com ventilação natural os ruídos externos foram levemente superiores ou iguais aos internos (índices $\mathrm{I} / \mathrm{E} \leq 1,0$ ).

Para facilitar a comparação dos resultados com os limites estabelecidos pela NBR 10.152/1987 da ABNT, os níveis médios de cada amostragem foram ilustrados. A Figura 2a apresenta as médias aritméticas e desvios-padrão (barras de erros) dos dados internos destacando a faixa adequada para interiores de salas de aula (40 a $50 \mathrm{~dB}$ ), enquanto a Figura $2 \mathrm{~b}$ traz os dados externos e sua respectiva faixa limítrofe (45 a $55 \mathrm{~dB})$. 


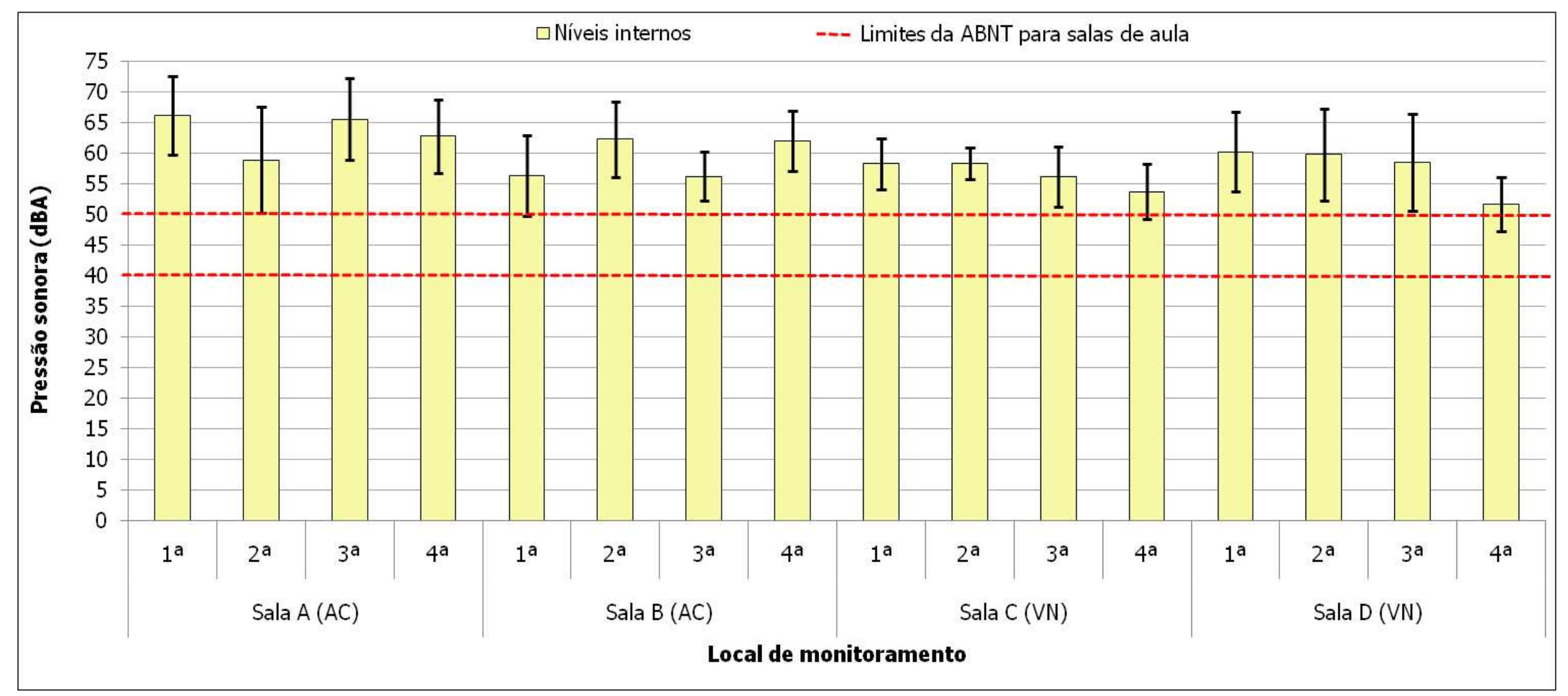

FIGURA 2a - Níveis internos de ruídos monitorados na UFSCar. 




FIGURA 2b - Níveis externos de ruídos monitorados na UFSCar. 
Na Figura 2a verifica-se que os níveis internos de ruídos excederam o valor máximo $(50 \mathrm{~dB})$ tolerado pela $\mathrm{ABNT}$ em todas as amostragens. Nos exteriores (Figura $2 b$ ) das salas de ventilação natural os ruídos também ultrapassaram o limite (55 dB) em 100\% das amostragens, enquanto para os exteriores das salas climatizadas o mesmo ocorreu em $50 \%$ dos monitoramentos.

Conforme citado, nos interiores climatizados os altos níveis são resultantes do ruído gerado devido ao funcionamento do aparelho de ar-condicionado, portanto uma importante medida a ser tomada seria a constante manutenção destes equipamentos para que nenhuma irregularidade prejudique sua operação e origine níveis maiores de ruídos. É importante destacar que a interferência externa do arcondicionado foi menor, pois o decibelímetro era mantido a certa distância do compressor do equipamento (que conforme citado eram do tipo split piso-teto).

Neste contexto, outra informação importante sobre a utilização do arcondicionado nas salas avaliadas é que em ambas eles funcionaram no modo de refrigeração, com a temperatura, definida pelos professores, oscilando entre $22 \mathrm{e}$

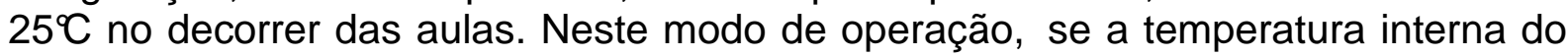
ambiente estiver acima da temperatura selecionada, o compressor do aparelho é ativado, em caso contrário, o compressor permanece desativado, porém a ventilação interna continuará operando (GREE, 2016). Assim, quando o compressor permanece ativado, os níveis de ruídos externos são maiores nas proximidades da unidade externa do ar-condicionado.

O fato supracitado comprova que a preocupação com a temperatura selecionada para o ar-condicionado não se limita a obtenção do conforto térmico, mas também interfere no conforto acústico dos ocupantes do ambiente. Um estudo realizado em salas de aula que possuíam meios mecânicos de ventilação comprovou que os altos níveis de ruídos gerados implicavam em correlações positivas com a intensidade vocal dos professores (GUIDINI et al., 2012). Em decorrência da elevação do tom de voz de professores, em resposta ao aumento do ruído ambiental, casos de disfonia durante o exercício profissional são frequentemente diagnosticados.

Em relação aos interiores ventilados naturalmente, avaliados no presente estudo, foi notória a contribuição dos ruídos externos, pois, além dos índices I/E menores ou iguais a 1,0, a manutenção de portas e janelas abertas contribuía para este cenário. Nos arredores das salas de aula circulavam muitos alunos e o ruído gerado externamente (pelas conversas e movimentação de pessoas e automóveis) afetava o conforto acústico dos ocupantes das salas. Neste contexto, quando viável, as portas poderiam ser mantidas fechadas ao menos nos horários de pico da circulação de alunos (especialmente próximos aos intervalos entre aulas) e seria interessante uma campanha de conscientização sobre os malefícios à saúde humana decorrente da poluição sonora, para que as pessoas se policiassem quanto à emissão de altos níveis de ruídos.

Campanhas de conscientização, como a sugerida, são almejadas por entidades de todo o mundo há décadas e culminaram na criação de um evento para abordar este tema: o Dia Internacional da Conscientização sobre o Ruído (International Noise Awareness Day - INAD), que ocorre desde 1996 (sempre na última quarta-feira do mês de abril) e objetiva conscientizar a população sobre o ruído e seus efeitos na saúde, na qualidade de vida, no meio ambiente e, também, sobre a responsabilidade de cada cidadão em reduzir o ruído gerado pelas atividades diárias (CENTER FOR HEARING AND COMMUNICATION, 2016). Além disso, estudos realizados em ambientes escolares concluíram que mudanças na 
atitude dos alunos em relação às conversas resultaram em grandes reduções nos níveis de ruídos (SERVILHA; DELATTI, 2014).

É interessante ressaltar que em ambientes escolares os níveis de ruídos toleráveis pela ABNT são mais baixos do que aqueles previstos para outros tipos de interiores. Por exemplo, a NR 15 do Ministério do Trabalho e Emprego (BRASIL, 1978), em seu Anexo 1, estipula que para níveis de ruído contínuo ou intermitente de até $85 \mathrm{dBA}$, o tempo de exposição máxima diária é de 8 horas. Apenas para critérios de comparação, na Figura $2 a$ verifica-se que todos os valores foram inferiores a $85 \mathrm{dBA}$. Porém, mesmo níveis abaixo deste padrão, podem interferir na comunicação humana e causar irritabilidade, além disso, por exemplo, ruídos na faixa entre 50 e $60 \mathrm{~dB}$ são suficientes para interferir na qualidade do sono de uma pessoa, provocando sensação de fadiga ao acordar (BOTKIN \& KELLER, 2011).

\section{CONCLUSÃO}

A poluição sonora existe em níveis elevados nas salas de aula avaliadas da Universidade Federal de São Carlos, pois os ruídos excederam o limite estipulado pela NBR 10.152/1987 da ABNT.

Nas salas de aula que possuem ventilação natural a principal fonte geradora de ruídos foi a circulação externa de pessoas, enquanto para as salas climatizadas foi o ruído do próprio funcionamento do ar-condicionado que mais contribuiu para os níveis elevados. Neste contexto, algumas medidas corretivas e preventivas incluem desde a manutenção periódica do equipamento de ar condicionado até a conscientização da comunidade acadêmica sobre os malefícios dos altos níveis de ruídos na saúde humana, para tentar mitigar aqueles gerados pelos transeuntes nos arredores das salas.

Este estudo, além dos alertas supracitados, contribuiu para o histórico de pesquisas sobre poluição sonora na cidade de São Carlos, indicando que mais um ambiente escolar encontra-se em desacordo com os limites legislados pela ABNT e que seria fundamental a ocorrência de fiscalizações periódicas para possibilitar a redução efetiva dos níveis de ruídos.

\section{REFERÊNCIAS}

ASSOCIAÇÃO BRASILEIRA DE NORMAS TÉCNICAS (ABNT). NBR 6401: instalações centrais de ar-condicionado para conforto - parâmetros básicos de projeto. Rio de Janeiro, 1980. $17 \mathrm{p}$.

NBR 10151: avaliação do ruído em áreas habitadas visando o conforto da comunidade - procedimento. Rio de Janeiro, 2000. 4 p.

NBR 10152: níveis de ruído para conforto acústico. Rio de Janeiro, 1987. 4 p.

BOTKIN, D. B.; KELLER, E. A. Ciência Ambiental: Terra, um planeta vivo. 7.ed. Rio de Janeiro: LTC, 2011. 681 p.

BRASIL. Ministério do Trabalho e Emprego. Portaria GM nํ 3214, de 8 de junho de 1978. NR 15 - Atividades e operações insalubres. Normas Regulamentadoras de Segurança e Saúde no trabalho. Brasília, 1978. 85 p. 
CENTER FOR HEARING AND COMMUNICATION (CHC). International Noise Awareness Day (INAD). Disponível em: <http://chchearing.org/noise/day/>. Acesso em: 09 fev. 2016.

COMIN, T. T. Monitoramento ambiental em uma escola da cidade de São Carlos-SP: ênfase em qualidade do ar interior. Disponível em: <http://www.bdtd.ufscar.br/htdocs/tedeSimplificado//tde_busca/arquivo.php?codArqui vo=4946>. Acesso em: 8 fev. 2016.

ENVIRONMENTAL PROTECTION AGENCY (EPA). Noise Pollution. Disponível em: <http://www.epa.gov/clean-air-act-overview/title-iv-noise-pollution>. Acesso em: 22 jan. 2016.

GREE. Condicionador de ar piso/teto - Manual do usuário, modelo GST 36/A. Disponível em: <http://pdf.webarcondicionado.com.br/gree/manual/usuario/mdupisoteto-gst24le-gst36la-gst42la-gst60la.pdf>. Acesso em: 09 fev. 2016.

GUIDINI, R. F. et al. Correlations between classroom environmental noise and teacher's voice. Revista da Sociedade Brasileira de Fonoaudiologia, v. 17, n. 2, p. 398-404, 2012. Disponível em: <http://dx.doi.org/10.1590/S151680342012000400006>. doi: 10.1590/S1516-80342012000400006.

SERVILHA, E. A. M.; DELATTI, M. A. College students' perception of classroom noise and its consequences on learning quality, Audiology Communication Research, v.19, n. 2, p. 138-144, 2014. Disponível em: <http://dx.doi.org/10.1590/S2317-64312014000200007>. doi: 10.1590/S231764312014000200007.

SILVA, F. P. et al. Avaliação de impacto ambiental: análise dos indicadores socioambientais na implantação do veículo leve sobre trilhos. Revista Eletrônica Interdisciplinar, v. 2, n. 14, p. 72-78, 2015. Disponível em: $<$ http://revista.univar.edu.br/index.php/interdisciplinar/article/view/431/360>. Acesso em: 8 fev. 2016.

TORRES, M. T. et al. Health and well-being in urban environment: from policies to pratice. Revista Portuguesa de Saúde Pública, v. 31, n. 1, p. 95-107, 2013. Disponível em: <http://dx.doi.org/10.1016/j.rpsp.2013.04.001>. doi: 10.1016/j.rpsp.2013.04.001. 